\title{
The comparison of endoscopic and external surgical approaches for sinonasal inverted papilloma and their impact on tumour recurrence
}

\author{
Zuzanna Nowinka, 3rd Year Student, Shadaba Ahmed FRCS, ORL-HNS
}

\begin{abstract}
Introduction: Sinonasal inverted papilloma (SNIP) is a rare, benign tumour originating from the mucosal lining of the sinonasal cavity. The treatment is aimed at complete tumour resection. Current resection methods include external excision, endoscopic surgery and a combined approach. The high rate of SNIP recurrence poses a challenge for the ear nose and throat (ENT) specialist. There is a debate about which method provides the best outcomes. In this study, the most recent literature is analysed to evaluate the effectiveness of each surgical approach in terms of recurrence rate and surgical complications.
\end{abstract}

Results: The literature search yielded data for 941 patients from ten studies. The analysis showed a recurrence rate of $13.5 \%$ for the endoscopic approach, $34 \%$ for combined and $36.7 \%$ for the external. The lowest recurrence rate $(0 \%-3.6 \%)$ was reported when novel endoscopic approaches, such as prelacrimal recess or ' $T u N a$ ' saving, were used. However, the comparison of surgical complications was challenging due to inconsistent reporting.

Discussion: The external approach previously considered a 'gold standard' is now becoming replaced by endoscopic surgery. The advantages of an endoscopic approach are a lower recurrence rate, better visualisation of the tumour and a more precise excision. Moreover, this approach preserves the surrounding tissues, reducing the amount of complications and recovery time. However, the external approach should still be employed when SNIP is widespread or in proximity with vital structures. Careful pre-operative planning is needed as the primary surgery has the best chances of eliminating the disease. Each revision surgery subsequently increases the risk of SNIP recurrence. Furthermore, there is a need for agreement on minimal surveillance time after SNIP resection.

Conclusion: The endoscopic approach should be a mainstay of SNIP treatment due to low recurrence rate and better patient outcomes. Nevertheless, ENT specialists should keep in mind that for more advanced disease, external and combined methods may be more appropriate.

\section{INTRODUCTION}

\section{Background}

Sinonasal inverted papilloma (SNIP) is a rare benign tumour of the sinonasal cavity with an incidence of $0.6-$ $1.5 / 100,000$ per year. ${ }^{1}$ The name 'inverted' originates from the characteristic endophytic growth, in which the neoplastic epithelium invaginates into the underlying connecting tissue, without invading the basement membrane. ${ }^{2}$ There is a male preponderance with a male-tofemale ratio of 2-5:1, and the majority of patients diagnosed in their fifties. ${ }^{1,3}$ Several factors, including smoking and HPV infection, have been associated with SNIP but the tumour pathogenesis is yet to be understood..,2

The majority of SNIP tumours originate in the maxillary sinus and lateral nasal walls (Figure 1). ${ }^{2}$ The clinical features are non-specific and therefore a biopsy and a CT or MRI scan are required for the diagnosis. ${ }^{3}$ Unilateral nasal obstruction, rhinorrhoea, headache, hyposmia and epistaxis are classical SNIP symptoms. ${ }^{1}$ On endoscopic examination, the tumour is lobulated with a reddish-grey colour and bleeds easily when touched. ${ }^{3}$
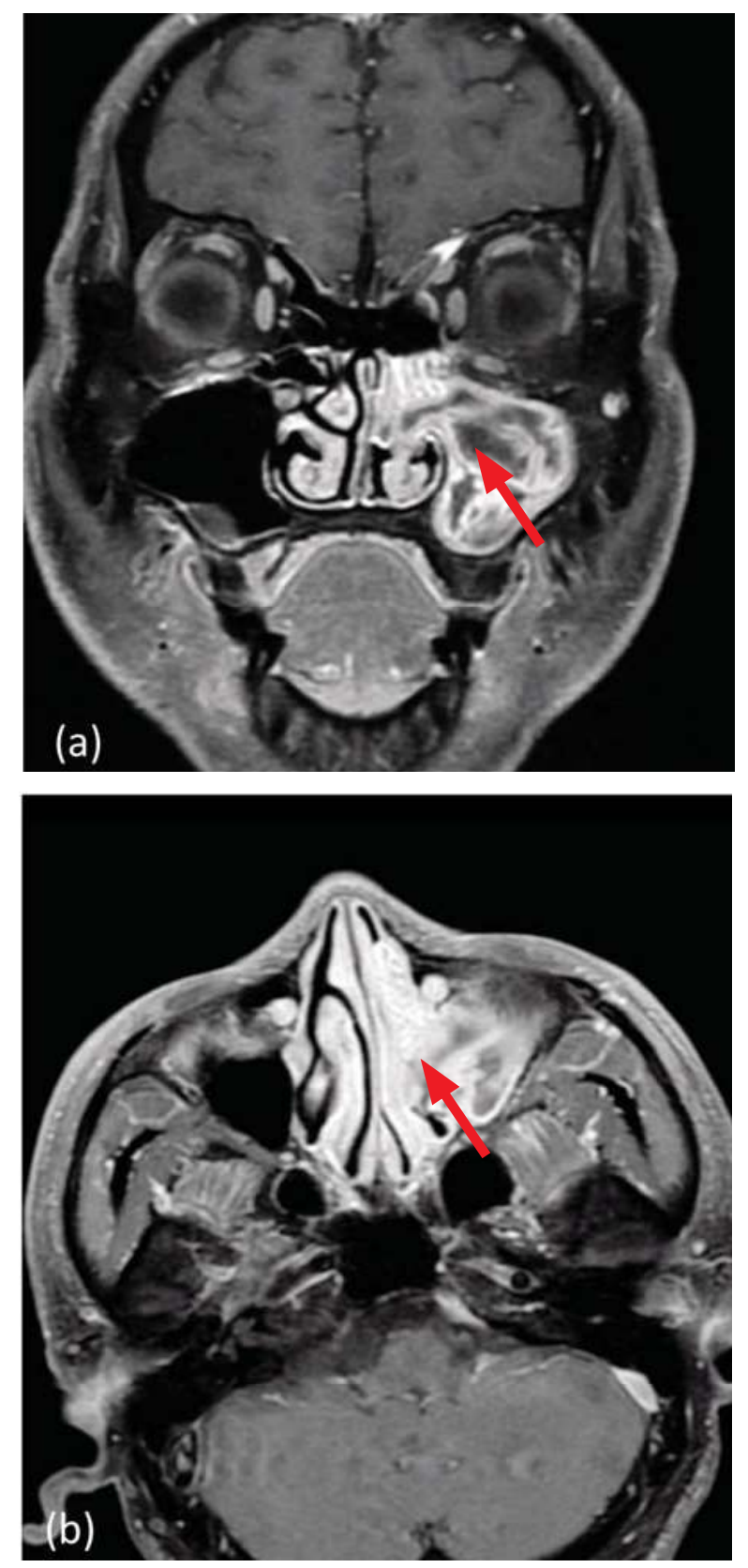

Figure 1: (a) MRI scan with contrast (coronal view) of SNIP involving left maxillary sinus (red arrow pointing towards inverted papilloma mass). ${ }^{4}$ (b) MRI scan of the same patient (sagittal view). ${ }^{4}$ 


\begin{tabular}{|c|l|}
\hline Stage & Characteristics \\
\hline T1 & $\begin{array}{l}\text { Tumour confined only to the nasal cavity, no extension into the sinuses. No associated } \\
\text { malignancy. }\end{array}$ \\
\hline T2 & $\begin{array}{l}\text { Tumour involving the ostiomeatal complex, and ethmoid sinuses, and/or the medial portion of } \\
\text { the maxillary sinus, with or without the involvement of the nasal cavity. No associated } \\
\text { malignancy. }\end{array}$ \\
\hline T3 & $\begin{array}{l}\text { Tumour involving the lateral, inferior, superior, anterior, or posterior walls of the maxillary sinus, } \\
\text { the sphenoid sinus, and/or the frontal sinus, with or without involvement of the medial portion } \\
\text { of the maxillary sinus, the ethmoid sinuses, or the nasal cavity. No associated malignancy. }\end{array}$ \\
\hline T4 & $\begin{array}{l}\text { All tumours with any extranasal/extrasinus extension to involve adjacent, contiguous structures } \\
\text { such as the orbit, the intracranial compartment or the pterygomaxillary space. All associated } \\
\text { with malignancy. }\end{array}$ \\
\hline
\end{tabular}

Table 1: Krouse classification of sinonasal inverted papilloma. ${ }^{5}$

Several staging systems have been described but the Krouse classification is most widely accepted (Table 1). ${ }^{5}$ The Krouse staging system is based on the tumour extensiveness and the presence of malignancy. It provides guidance for choosing the surgical method of removal.

\section{Current management of SNIP}

SNIPs are especially challenging for otolaryngologists due to their high recurrence rate and potential for malignant transformation. ${ }^{3}$ Following the surgical excision, SNIP will recur in around a quarter of cases and $5-15 \%$ of lesions will become cancerous. ${ }^{6}$ Recurrence often occurs at the site of the original tumour and is related to incomplete SNIP resection during primary surgery. ${ }^{3}$

The current guideline on 'Nose and paranasal sinus tumours' published in The Journal of Laryngology and Otology states that the SNIP treatment should be

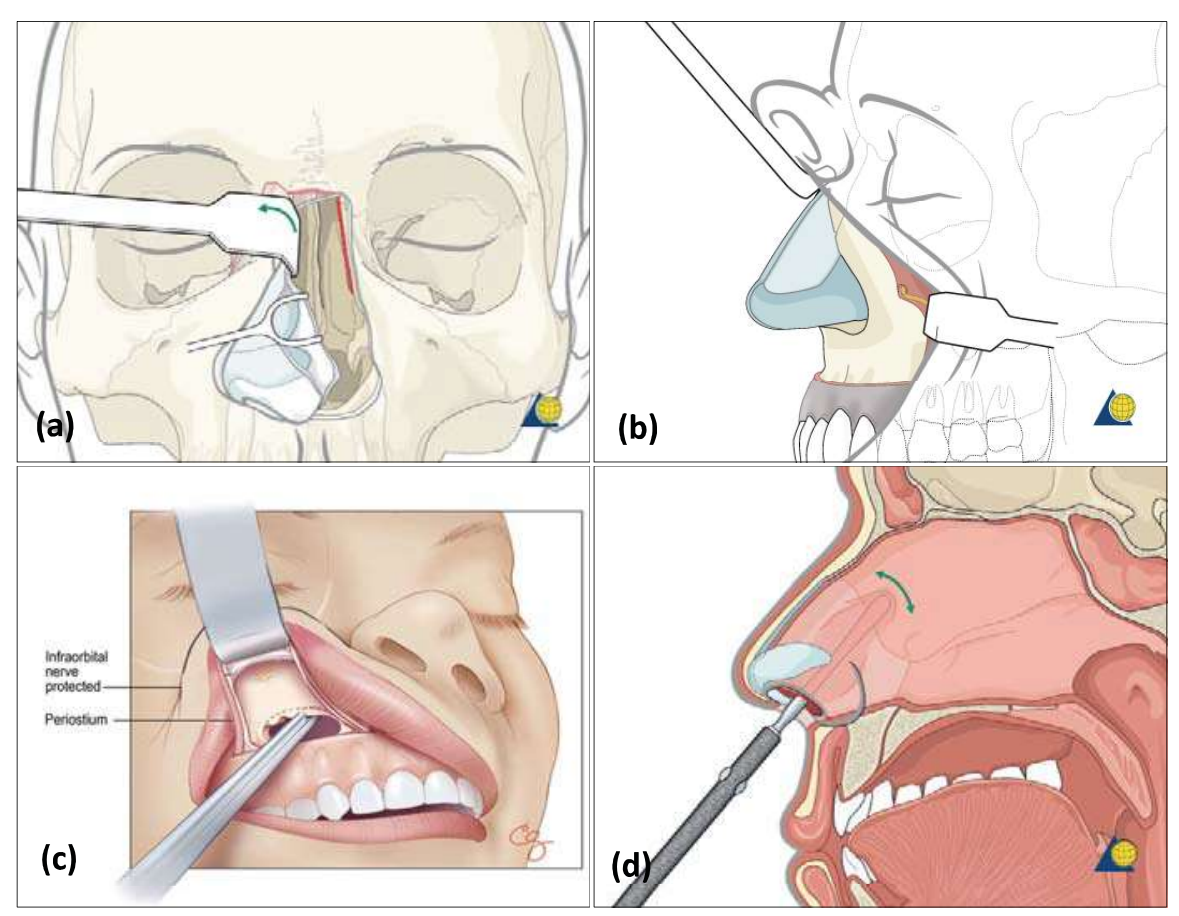

Figure 2: Different approaches to SNIP resection: (a) lateral rhinotomy (b) midfacial degloving (c) Caldwell Luc procedure (d) intranasal endoscopy. ${ }^{14-16}$ aimed at the complete surgical removal of the tumour while preserving the adjacent organs. The best outcome is achieved when the tumour is treated de novo, as each subsequent operation increases the risk of further recurrence, leading to more scarring and distorted anatomy of the sinonasal cavity. ${ }^{7,8}$ Therefore, there is a need for careful pre-operative planning and choosing an appropriate surgical approach according to the tumour size, site and origin.

Historically, SNIPs resection was achieved externally by a medial maxillectomy via lateral rhinotomy (Figure 2a). Subsequently, midfacial degloving (Figure 2b) was introduced as an alternative to minimise the extensive facial scarring caused by the traditional approach. ${ }^{9}$ This was then followed by less invasive external techniques such as Caldwell-Luc were developed (Figure 2c). Finally, in the 1990s, an intranasal endoscopic medial maxillectomy (Figure 2d) was developed by Waitz and since then become the most popular surgical method for SNIP treatment. ${ }^{10}$ Although effective, the endoscopic resection leads to complications such as epiphora, cerebrospinal fluid leakage or an inability to feel the nasal flow. Image-guided resection or preservation of the inferior turbinate are examples of novel endoscopic techniques, aimed at reducing the risk of surgical complications and SNIP recurrence. ${ }^{11-13}$

There is a high variation in the technique employed by surgeons as it remains uncertain which approach yields the best results and minimises the risk of SNIP recurrence. The argument favouring the external approach is that it provides wide access and good exposure of the sites with difficult entry, i.e. frontal sinuses. ${ }^{3}$ The endoscopic approach is more delicate to the surrounding structures and reduces the tissue damage ${ }^{17}$ but again, the tumour can be difficult to access and some lesions may be missed. ${ }^{18}$ In this case, a surgeon may decide to use a combined approach, where both external and intranasal techniques are used together. ${ }^{19}$ 


\section{Aims}

The two most recent systematic reviews concluded that there is a growing trend towards endoscopic surgery ${ }^{1}$ as this approach may decrease the recurrence rate of SNIP. ${ }^{18}$ However, the studies published after 2013 were not included in these reviews and the incidence of surgical complications was not evaluated. Although unrelated to the tumour recurrence, the complications can negatively affect the patient's quality of life.

Therefore, the aim of this systematic review is to analyse the most recent literature regarding the surgical treatment of SNIPs. All surgical approaches will be reviewed, including studies outlining novel endoscopic techniques. The following research question will be answered: "What is the current most effective surgical approach for SNIP in terms of the recurrence rate and surgical complications?"

\section{RESULTS}

\section{General findings}

Ten studies were selected and analysed in this review. ${ }^{8,11-13,19-24}$ Nine of them were retrospective cohorts and one was a prospective cohort study. ${ }^{21}$ Seven studies compared the external to endoscopic approach and three investigated the use of modified approaches. ${ }^{11-13}$

The literature search yielded 941 patients with a 2.2:1 male-to-female ratio. The average age of disease onset was 55 years, with the youngest case being 17 years old and the oldest 89 , which is comparable to SNIP epidemiology described in the literature. ${ }^{1,3}$ As shown in Table 2 nearly $60 \% \quad(n=506)$ of patients had a primary IP and the remaining cases had undergone revision surgery.
Subsequently, four studies excluded 117 cases due to: associated malignancy, ${ }^{8,20}$ too short follow-up period, ${ }^{20,23}$ inaccurate diagnosis ${ }^{23}$ or bilateral frontal brain lobes involvement. ${ }^{21}$ In four of the ten case series, only patients with maxillary sinus disease were included.11, 12, 19, 24

Table 3 represents the Krouse classification, which was assessed in six studies. ${ }^{8,12,13,20,22,23}$ Out of 712 cases, the majority were T2 (40\%) and T3 (43\%) stages. One case series subsequently excluded all $\mathrm{T} 4$ stage patients from the analysis due to associated malignancy. ${ }^{8}$

\section{Surgical approach}

The average follow-up of the cases varied from 24 months to 8.8 years. One study had an individual observation period of six months, despite the average follow-up of two years. ${ }^{22}$ Table 4 below provides a summary of the findings on SNIP recurrence according to the surgical approach.

The total SNIP recurrence rate for all surgical approaches was $19.5 \%(n=161)$. There was a general trend towards the endoscopic approach, which was performed in $73 \%$ of cases $(n=602)$. Only $13.5 \%(n=81)$ of these patients in this group had tumour recurrence. For the maxillary sinus disease, the transnasal medial maxillectomy was the most commonly employed endoscopic approach. . $^{8,13,19-21,24}$ Besides, a great variation in the used technique was observed. Some studies describe the use of 'frozen sections', which is a form of an intraoperative, rapid macroscopic analysis used to determine the margins of a tumour. ${ }^{12,20,24}$ Other have used the 'pedicle-oriented endoscopic surgery', focused at resecting the attachments of the tumour and drilling the underlying bone at the site of tumour origin. ${ }^{11}$

The traditional external resection of SNIP was adopted to treat only $20 \%$ of patients $(n=169)$. 'This surgical method was employed in three studies and consisted of midfacial degloving and lateral rhinotomy. ${ }^{8,22,23}$

\begin{tabular}{|c|c|c|c|c|c|c|}
\hline Study & $\begin{array}{c}\text { Initial patient } \\
\text { population }\end{array}$ & Mean age & Males & Females & Primary cases & Revision cases \\
\hline Sciarretta V. (2014) & 141 & 58.7 & 101 & 40 & 87 & 54 \\
\hline Kamel R. (2014) & 51 & 49.0 & 34 & 17 & 18 & 33 \\
\hline Kadapa N. (2014) & 28 & 46.07 & 20 & 8 & 28 & 0 \\
\hline Nygren A. (2016) & 88 & 58.0 & 56 & 32 & - & - \\
\hline Zydron R. (2016) & 69 & 55.6 & 41 & 28 & 79 & 20 \\
\hline Bugter O. (2017) & 323 & 55.8 & 245 & 78 & 121 & 19 \\
\hline Pagella F. (2017) & 84 & 66.6 & 53 & 11 & 18 & 10 \\
\hline Wu V. (2018) & 28 & 54.9 & 17 & 25 & 58 & 0 \\
\hline Ahn S. (2018) & 58 & 53.8 & 33 & 27 & 42 & 29 \\
\hline Yu QQ. (2018) & 71 & 52.0 & 44 & 297 & 506 & 347 \\
\hline Total & 941 & 55.0 & $2.2: 1$ male-to-female ratio & $59.3 \%$ & $40.7 \%$ \\
\hline
\end{tabular}

Table 2: Patient's characteristics. ${ }^{8,11-13,19-24}$

\begin{tabular}{|c|c|c|}
\hline Krouse stage & Patients & $\%$ \\
\hline T1 & 71 & 10 \\
\hline T2 & 288 & 40 \\
\hline T3 & 306 & 43 \\
\hline T4 & 47 & 7 \\
\hline
\end{tabular}

Table 3: Classification of patients by Krouse staging system. ${ }^{8,11-13,19-24}$ 
The comparison of endoscopic and external surgical approaches for sinonasal inverted papilloma and their impact on tumour recurrence Zuzanna Nowinka, Shadaba Ahmed

\begin{tabular}{|c|c|c|c|c|c|c|c|c|c|}
\hline \multirow[b]{2}{*}{ Study } & \multirow{2}{*}{$\begin{array}{c}\text { Total } \\
\text { number of } \\
\text { patients }\end{array}$} & \multirow{2}{*}{$\begin{array}{c}\text { Total } \\
\text { recurrence } \\
(\%)\end{array}$} & \multicolumn{2}{|c|}{ Endoscopic } & \multicolumn{2}{|c|}{ Combined } & \multicolumn{2}{|c|}{ External } & \multirow{2}{*}{$\begin{array}{l}\text { Follow up } \\
\text { (years) }\end{array}$} \\
\hline & & & Cases & $\begin{array}{l}\text { Recurrence } \\
\text { (\%) }\end{array}$ & Cases & $\begin{array}{c}\text { Recurrence } \\
(\%)\end{array}$ & Cases & $\begin{array}{c}\text { Recurrence } \\
(\%)\end{array}$ & \\
\hline Sciarretta V. (2014) & 110 & 6.4 & 110 & 6.4 & - & - & - & - & 4.7 \\
\hline Kamel R. (2014) & 51 & 15.7 & 51 & 15.7 & - & - & - & - & 8.8 \\
\hline Kadapa N. (2014) & 27 & 22.2 & 14 & 21.4 & 13 & 23.1 & - & - & 3.4 \\
\hline Nygren A. (2016) & 79 & 25.3 & 60 & 23.3 & 10 & 10.0 & 9 & 55.6 & 4.5 \\
\hline Zydron R. (2016) & 69 & 40.6 & 47 & 31.9 & 16 & 62.5 & 6 & 50.0 & 4.7 \\
\hline Bugter O. (2017) & 247 & 30.0 & 79 & 20.3 & 14 & 28.6 & 154 & 35.1 & 5.5 \\
\hline Pagella F. (2017) & 84 & 3.6 & 84 & 3.6 & - & - & - & - & $>2$ \\
\hline Wu V. (2018) & 28 & 0.0 & 28 & 0 & - & - & - & - & 2.5 \\
\hline Ahn S. (2018) & 58 & 15.5 & 58 & 15.5 & - & - & - & - & 2.1 \\
\hline Yu QQ. (2018) & 71 & 8.5 & 71 & 8.5 & - & - & - & - & 5.5 \\
\hline Total & 824 & $19.5 \%$ & 602 & $13.5 \%$ & 53 & $34.0 \%$ & 169 & $36.7 \%$ & \\
\hline
\end{tabular}

Table 4: Summary of recurrence data according to the surgical approach., 11-13, 19-24

\begin{tabular}{|c|c|c|c|c|c|c|}
\hline \multirow{2}{*}{ Study } & \multirow{2}{*}{ Patients } & \multicolumn{2}{|c|}{ Intervention } & \multicolumn{2}{|c|}{ Control } & \multirow{2}{*}{ Follow up } \\
\cline { 3 - 6 } & & Cases & Recurrence & Cases & Recurrence & \\
\hline Pagella F. (2017) & 84 & 29 & 0 & 55 & 3 & $>24$ months \\
\hline Yu QQ. (2018) & 71 & 20 & 1 & 51 & 5 & 5.5 years \\
\hline Ahn S. (2018) & 58 & 34 & 2 & 24 & 7 & 25 months \\
\hline Total & 213 & 83 & $3(3.6 \%)$ & 130 & $15(11.5 \%)$ & \\
\hline
\end{tabular}

Table 5: SNIP recurrence in newer endoscopic approaches. ${ }^{11-13}$

In this patient population, the rate of tumour recurrence was on average three times higher $(36.7 \%)$ than in the endoscopic group (13.5\%).

The combined approach, usually involving the Caldwell-Luc incision with endoscopic assistance, was used in $6 \%$ of cases $(n=53)$. A third of patients $(n=18)$ had SNIP recurrence, much higher than in the endoscopic group (13.5\%). However, the recurrence was only slightly lower than in the external resection group (36.7\%). This approach was generally indicated for cases with widespread SNIPs or involving frontal sinuses, lateral walls of maxillary sinus or in close proximity with the orbit. ${ }^{20,21}$

\section{Novel endoscopic techniques}

Treating SNIP with novel endoscopic approaches and navigation technology leads to quicker recovery and fewer complications, with a reduced rate of tumour recurrence. ${ }^{11-13}$ Table 5 summarises the recurrence data from these studies.

The 'TuNa' saving approach was developed to avoid common surgical complications like lacrimal stenosis. This procedure spares the inferior turbinate and nasolacrimal duct, so that the lacrimal pathway is protected. None of the patients operated upon with this approach had a recurrence of SNIP or complications. ${ }^{11}$

In 'prelacrimal recess' approach (PRLA) the maxillary sinus is accessed through the prelacrimal recess rather than via middle meatus antrum. ${ }^{12}$ This method was developed to replace the Caldwell-Luc incision as it provides access to the anterior wall of maxillary sinus without damaging the nasolacrimal duct.

An image-guided navigation system allows for the calibration of the CT images to the patient's head anatomy. As a result, the surgeon can localise the tumour and important structures during the endoscopic sinus surgery. This approach significantly lowered the SNIP recurrence rate compared to the standard surgery without navigation system. ${ }^{13}$
The above methods were shown to be safer and more effective than the standard endoscopic intranasal medial maxillectomy. As represented in Table 5, treating patients with the novel approaches described decreases the SNIP recurrence rate when compared to the standard endoscopic approach by more than double.

\section{Surgical complications}

The surgical complications were generally not well reported as the authors either did not specify the surgical approach $^{23}$ or describe the type of complication. ${ }^{22}$ The highest observed incidence of complications was $15 \%$, but this study considered patients treated with endoscopy and externally together. ${ }^{23}$ Reported problems were: 'leakage of cerebrospinal fluid, wound infections, epiphora, meningitis, death'.

With regards to the endoscopic approach, the most commonly reported complications were: epistaxis, epiphora, mucocele of the sinus and leakage of cerebrospinal fluid. ${ }^{11-13,20}$ These problems seem to be addressed by the newer endoscopic techniques, as none were observed in these patient groups. ${ }^{11-13}$ Additionally, patients treated via the endoscopic approach had on average a significantly shorter length of hospital stay (2.83 days) than the patients operated upon with external resection (4.68 days). ${ }^{22}$

\section{DISCUSSION}

\section{Surgical approach and SNIP recurrence}

The increasingly popular endoscopic surgery is currently the most effective method of SNIP resection and should be considered a mainstay treatment. ${ }^{25}$ This review showed that the recurrence rate after endoscopic resection is nearly three times lower than after other approaches (Table 4). Additionally, this approach avoids extensive facial incisions, which decreases the degree of facial oedema and scarring. Quicker recovery and shorter length 


\section{The comparison of endoscopic and external surgical approaches for sinonasal inverted papilloma and their impact on tumour recurrence Zuzanna Nowinka, Shadaba Ahmed}

of hospital stay are other advantages of this method. Novel endoscopic approaches to SNIP resection give even more promising results in terms of low recurrence rate and low risk of surgical complications (Table 5). Considering the advancing technology, the external and combined surgeries seem quite outdated.

The recurrence of SNIP is thought to be a reappearance of residual disease that was inadequately resected. ${ }^{3}$ Thanks to modern magnifying systems, the endoscopic surgery provides a better visualisation of the tumour. Enhanced view of the nasal cavity enables to distinguish a diseased mucosa from a healthy one, which subsequently allows for a more precise tumour clearance. ${ }^{22}$ Likewise, studies reporting the use of 'frozen sections' $12,20,24$ or attachment-oriented tumour resection ${ }^{11,20}$ reported a low recurrence rate. These techniques allow for a detailed resection of tumour margins as well as drilling the underlying bone at attachment sites, ensuring no residual disease is left. ${ }^{11}$

Similarly, novel endoscopic techniques, such as imageguided excision or prelacrimal recess approach (PRLA) approach, enhance the exploration and navigation within the nasal cavity, allowing for more careful excision of the tumour while preserving important surrounding structures. ${ }^{12,13}$ Low recurrence rate and no surgical complications were reported, meaning that these modifications are both effective and safe. ${ }^{11-13}$ However, these studies had several limitations such as relatively small patient cohort and short observation period. ${ }^{11-13}$ Even though SNIP is a rare condition, a larger study group with longer follow-up is required to state the superiority of one management method over another. Besides, all of the analysed studies show a high variation in surgical technique employed, making the comparison of outcomes rather difficult. Future research should aim to outline the resection technique in more detail to allow the objective comparison.

Previously considered a gold standard treatment for SNIP, the external approach is now becoming less popular. In this review, only three out of ten studies have operated using this resection method. ${ }^{8,22,23}$ A high recurrence rate of nearly $40 \%$ (Table 4) as well as associated extensive facial scarring, swelling and painful recovery, explain why this approach is becoming less favourable than the endoscopic resection. Furthermore, the external approach distorts the important anatomical landmarks, leads to the creation of scar tissue and adhesions within the nasal cavity, making any further revision surgery more challenging. ${ }^{19}$ These difficulties can be tackled when a primary surgery is carried out via less invasive endoscopic approach, ${ }^{19}$ in which more delicate handlings prevents the damage to the surrounding structures.

Nevertheless, the external approach should be still kept as an option for more widespread or highly recurrent cases. Some tumours may be simply too large or difficult to access via an endoscope (i.e. in close proximity with the orbit) and in these cases, the external approach should be adopted. ${ }^{20}$ The fact that the external approach is used for challenging and advanced cases ${ }^{3}$ can partially explain high recurrence rate in this patient group.

Therefore, the external approach can also be used when the operating surgeon is not skilful in endoscopic surgery. ${ }^{22}$, ${ }^{26}$ One study have observed that the recurrence rate has fallen in more recent surgeries ${ }^{22}$ and similarly, a study in which all operations were conducted by a single, qualified surgeon had very low recurrence rate. ${ }^{11}$ These findings prove that the success of endoscopic SNIP resection depends mainly on the surgeon's competency and knowledge of nasal anatomy. As these factors are likely to improve with time, the recurrence rate of SNIP will fall, as demonstrated by the surgical 'learning curve'. ${ }^{17,22}$

\section{Other factors influencing SNIP recurrence}

Patients undergoing a revision surgery are at higher risk of SNIP recurrence, regardless of the surgical approach. ${ }^{7,8,25}$ In this review, studies with a higher proportion of revision cases had subsequently a higher recurrence rate. ${ }^{19,23}$ It is possible that the choice of a primary surgery influences the risk of recurrence the most. Each operation leads to increased anatomical distortion and scar formation, making adequate tumour resection difficult. ${ }^{20}$ The number of previous revision surgeries is positively linked to increased risk of further recurrence, ${ }^{19,20}$ and therefore, the complete clearance at first surgery is the strongest predictor of successful SNIP treatment.

The average follow-up time varied greatly between the studies and ranged from 24 months to 8.8 years. ${ }^{11,19}$ Two studies with the lowest recurrence rate of SIP had a follow-up of 2-3 years ${ }^{11,24}$ and case series with longer surveillance (4-5 years) reported a higher overall recurrence rate. ${ }^{8,22,23}$ As the majority of SNIP cases recur within first two years post-surgery, most authors adopt that observation time in their studies. ${ }^{27}$ However, it has to be noted that with short follow-up, some cases will be missed, resulting in inaccurately reported recurrence rate. A monitoring time of 3-5 years has been suggested, and there is a debate whether the patients should be followed up for life due to malignant potential of SNIP. ${ }^{3}$ Yet, this may not be costeffective considering a low risk $(5 \%)$ of SNIP cancerous transformation. ${ }^{27} \mathrm{~A}$ consensus is needed on how long the patients should be followed post-surgery.

The Krouse staging seems to be unrelated to recurrence rate. ${ }^{26}$ Based on the findings of this study, it was impossible to observe any correlation between the Krouse stage and the recurrence rate. This shows the need for a better staging system which could help the clinicians identifying patients at highest risk of SNIP recurrence and determining appropriate follow-up time. It has been suggested tumour attachment site rather than tumour extensiveness is a better factor of predicting recurrence - for example tumours involving maxillary sinus show a higher tendency to recur. ${ }^{26}$ Nevertheless, Krouse classification may be helpful in preoperative planning and choosing the surgical approach since it is based on the extent of the disease.

\section{Surgical complications}

The surgical complications were inconsistently reported in the studies, which made the evaluation of the surgical complications challenging. Some authors stated the number but not described the complication ${ }^{22}$ or they did not specify in which patient group the problem has occurred. ${ }^{23}$ The lack of consistent reporting may stem from the fact that the studies focus primarily on SNIP recurrence rate and the surgical complications are usually not considered as a primary outcome. Nevertheless, the surgical complications are an important point to consider in future research. Better reporting of postoperative complications will allow to holistically evaluate the impact of surgery on patients' quality of life. 


\section{The comparison of endoscopic and external surgical approaches for sinonasal inverted papilloma and their impact on tumour recurrence Zuzanna Nowinka, Shadaba Ahmed}

\section{CONCLUSION}

Despite the advances in technology, the management of SNIP still poses a challenge for ENT specialists. As this systematic review has shown, there is a high risk of recurrence no matter which surgical approach is chosen. On average, a fifth of patients will require further surgery.

The endoscopic approach was shown to be the most effective method of SNIP resection. Better tumour visualisation, less facial scarring and quicker recovery are major advantages of this method. Novel endoscopic approaches showed particularly promising results; however, more evidence is needed. The endoscopic surgery should be also advised as a primary surgery due to less distortion to surrounding anatomy.

However, the treatment should be tailored to the individual. To reduce the recurrence and surgical complications, the surgical approach has to be chosen according to the location and extent of the lesion. The external and combined approaches should be reserved for tumours that would be inoperable with intranasal endoscopy because of their difficult location.

Future research should include detailed descriptions of the surgical technique, complications and follow-up to objectively evaluate the effectiveness of the surgical method. Krouse staging seems to be unrelated to SNIP recurrence and thus a better staging system is required. A consensus is also needed on the length of surveillance of SNIP patients.

\section{REFERENCES}

1. Attlmayr B, Derbyshire SG, Kasbekar AV, Swift AC. Management of inverted papilloma: review. J Laryngol Otol 2017;131(4):284-9.

2. Wang M-J, Noel JE. Etiology of sinonasal inverted papilloma: a narrative review. World J Otorhinolaryngol - Head Neck Surg 2017;3(1):54-8.

3. Lisan Q, Laccourreye O, Bonfils P. Sinonasal inverted papilloma: from diagnosis to treatment. Eur Ann Otorhinolaryngol Head Neck Dis 2016;133(5):337-41.

4. Radiopaedia.org. Inverted papilloma. Available from: https://radiopaedia.org/cases/inverted-papilloma5? lang $=\mathrm{gb}$ (accessed 30.6.19)

5. Krouse JH. Development of a staging system for inverted papilloma. Laryngoscope 2000;110(6):965-8.

6. Ungari C, Riccardi E, Reale G, Agrillo A, Rinna C, Mitro $\mathrm{V}$, et al. Management and treatment of sinonasal inverted papilloma. Ann Stomatol (Roma) 2015;6(3-4):87-90.

7. Lund VJ, Clarke PM, Swift AC, McGarry GW, Kerawala C, Carnell D. Nose and paranasal sinus tumours: United Kingdom National Multidisciplinary Guidelines. J Laryngol Otol 2016;130(S2):S111-S118. Available from: https://www.ncbi.nlm.nih.gov/pubmed/27841122 (accessed 30.6.19).

8. Nygren A, Kiss K, von Buchwald C, Bilde A. Rate of recurrence and malignant transformation in 88 cases with inverted papilloma between 1998-2008. Acta Otolaryngol 2016;136(3):333-6.

9. Kim HJ, Kim C-H, Kang J-W, Shin W-C, Kim YS, Do Y-K, et al. A modified midfacial degloving approach for the treatment of unilateral paranasal sinus tumours. J Cranio-Maxillofacial Surg 2011;39:284-8.

10. Waitz G, Wigand ME. Results of endoscopic sinus surgery for the treatment of inverted papillomas. Laryngoscope 1992;102(8):917-22.

11. Pagella F, Pusateri A, Matti E, Avato I, Zaccari D, Emanuelli E, et al. "TuNa-saving" endoscopic medial maxillectomy: a surgical technique for maxillary inverted papilloma. Eur Arch Oto-Rhino-Laryngolog 2017;274(7):2785-91.

12. Yu Q-Q, Guan G, Zhang N-K, Zhang X-W, Jiang Y, Lian Y-Y, et al. Intranasal endoscopic prelacrimal recess approach for maxillary sinus inverted papilloma. Eur Arch Oto-Rhino-Laryngology 2018;275(9):2297-302.

13. Ahn SH, Lee EJ, Kim JW, Baek KH, Cho H-J, Yoon J-H, et al. Better surgical outcome by image-guided navigation system in endoscopic removal of sinonasal inverted papilloma. J Craniomaxillofac Surg 2018;46(6):937-41.

14. Approach - Lateral Rhinotomy - AO Surgery Reference [Internet]. [cited 2019 Apr 8]. Available from: https:// www2.aofoundation.org/wps/portal/surgery.

15. Midface - Approach - Approaches to the maxilla - AO Surgery Reference [Internet]. [cited 2019 Apr 8]. Available from: https://www2.aofoundation.org/wps/ portal/surgery.

16. Medical Illustration Gallery [Internet]. [cited 2019 Apr 8]. Available from: http://chrisgralapp.com/medical. html.

17. Pai PS, Moiyadi A, Nair D. Endoscopic v/s conventional approach to sino-nasal tumours - What's the debate? Indian J Surg Oncol 2010;1(2):194-9.

18. Kim JS, Kwon SH. Recurrence of sinonasal inverted papilloma following surgical approach: a meta-analysis. Laryngoscope 2017;127(1):52-8.

19. Kamel RH, Abdel Fattah AF, Awad AG. Transnasal endoscopic medial maxillectomy in recurrent maxillary sinus inverted papilloma. Rhinology 2014;52(4):381-5.

20. Sciarretta V, Fernandez IJ, Farneti P, Pasquini E. Endoscopic and combined external-transnasal endoscopic approach for the treatment of inverted papilloma: analysis of 110 cases. Eur Arch Oto-RhinoLaryngology 2014;271(7):1953-9.

21. Kadapa NPB, Reddy LS, Reddy V, Kumuda P, Reddy MVV, Rao LMSCS. Nasal endoscope: an armamentarium in the management of sinonasal inverted papilloma. Indian J Otolaryngol Head Neck Surg 2014;66(2):200-4.

22. Zydroń R, Wierzbicka M, Greczka G. Clinical outcomes of treatment of sinonasal inverted papillomas (IPs) depending on the surgical technique and learning curve. Otolaryngol Pol = Polish Otolaryngol 2016;70(6):1-5.

23. Bugter O, Monserez DA, van Zijl FVWJ, Baatenburg de Jong RJ, Hardillo JA. Surgical management of inverted papilloma; a single-center analysis of 247 patients with long follow-up. J Otolaryngol - Head Neck Surg 2017;46(1):67.

24. Wu V, Siu J, Yip J, Lee JM. Endoscopic management of maxillary sinus inverted papilloma attachment sites to minimize disease recurrence. J Otolaryngol Head Neck Surg 2018;47(1):24.

25. Peng R, Thamboo A, Choby G, Ma Y, Zhou B, Hwang $\mathrm{PH}$. Outcomes of sinonasal inverted papilloma resection by surgical approach: an updated systematic review and meta-analysis. Int Forum Allergy Rhinol 2019;9(6):573581

26. Kim D-Y, Hong S-L, Lee CH, Jin H-R, Kang JM, Lee B-J, et al. Inverted papilloma of the nasal cavity and paranasal sinuses: a Korean multicenter study. Laryngoscope 2012;122(3):487-94.

27. Suh JD, Chiu AG. What are the surveillance recommendations following resection of sinonasal inverted papilloma? Laryngoscope 2014;124(9):1981-2.

Correspondence to: shadaba.ahmed@mbht.nhs.uk 